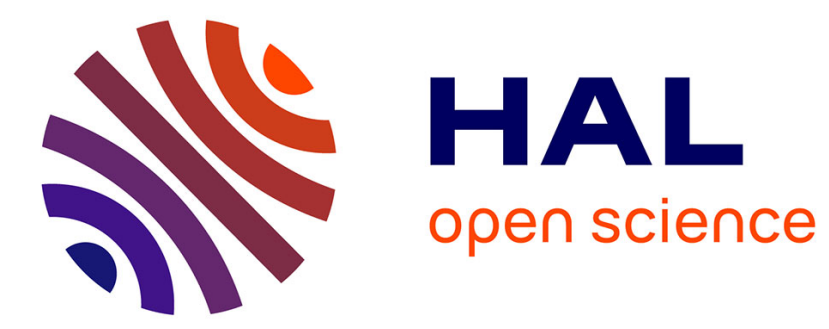

\title{
Le corps refoulé des chimistes
}

Bernadette Bensaude-Vincent

\section{To cite this version:}

Bernadette Bensaude-Vincent. Le corps refoulé des chimistes. La Lettre de la Maison Française d'Oxford, 1998, 9, pp.9-18. hal-02555969

\section{HAL Id: hal-02555969 \\ https://hal.science/hal-02555969}

Submitted on 27 Apr 2020

HAL is a multi-disciplinary open access archive for the deposit and dissemination of scientific research documents, whether they are published or not. The documents may come from teaching and research institutions in France or abroad, or from public or private research centers.
L'archive ouverte pluridisciplinaire HAL, est destinée au dépôt et à la diffusion de documents scientifiques de niveau recherche, publiés ou non, émanant des établissements d'enseignement et de recherche français ou étrangers, des laboratoires publics ou privés. 
La Lettre de la Maison Française

$n^{\circ} 9$, Hilary-Trinity Terms, pp.9-18

\section{Le corps refoulé des chimistes}

L

e titre de cette communication m'a été inspiré par mon travail sur la réforme de la nomenclature chimique opérée en 1787 par Guyton de Morveau, Lavoisier, Berthollet et Fourcroy. En effet, une comparaison des noms anciens et des noms nouveaux manifeste un refoulement massif des dénominations impliquant le corps. On sait que le principe de la nouvelle nomenclature est que chaque dénomination reflète la composition des substances et, dans bien des cas, la proportion des constituants. Ceci implique un double renoncement au corps. Se trouvent évacuées, premièrement, les références au corps comme cible d'action des substances, ou comme consommateur des substances, c'est-àdire les noms indiquant les vertus médicinales des substances chimiques. Le mot "émétique" doit disparaître au profit du "tartre de potasse antimoniale" et les "eaux hépatiques" deviennent "eaux sulfureuses" ou "sulfurées". Mais c'est avant tout sur l'évacuation des références aux qualités sensibles que je voudrais concentrer l'attention.

Sont chassés tous les noms évoquant l'aspect sensible des substances comme "beurre d'antimoine", "crème de tartre", "foie de soufre", "huile grasse" ou encore "les fleurs de benjoin" qui deviennent "acide benzoique sublimé". Eliminées les couleurs qui étaient, sans doute, les qualités les plus usitéees pour distinguer les sels au sein d'une espèce : par exemple les "couperoses" bleue, blanche ou verte deviennent respectivement "sulfates de zinc", de cuivre, ou de fer. Le "blanc de plomb" et le "bleu de Prusse" (utilisés en teinture) deviennent "oxyde de plomb" et "prussiate de fer". La vue n'était pas seule mobilisée: on parlait de "fluor pesant", de "mercure doux", "d'alcali caustique", ou d"air puant" lequel devient le "gaz hydrogène sulfuré".

Le langage de la "nouvelle chimie" se caractérise donc par l'exclusion du goût, de l'odorat, du toucher, bref des sens comme facultés de connaissance. Par contraste avec l'ancienne nomenclature qui désignait les corps par leur signification pour nous, par rapport à la connaissance qu'on en prend ou par rapport aux usages qu'on en fait, la nouvelle nomenclature construit l'objectivité substantielle en évacuant toute référence au corps sujet de savoir.

Cette mutation est solidaire d'un renversement des priorités entre données qualitatives et quantitatives. Comme l'a montré un excellent article 
de Lissa Roberts "The death of the Sensuous Chemist", à l'éducation sensorielle des chimistes proposée par Gabriel François Rouelle, à l'exercice $\mathrm{du}$ corps comme instrument d'analyse, Lavoisier substitue au Traité élementaire de chimie une éducation fondée sur l'analyse au sens mathématique, sur une langue analytique ${ }^{1}$. Certes, comme ne manque pas de le souligner Lissa Roberts, les chimistes continuent d'observer les changements de couleur, de sentir, de renifler leurs préparations ou les émanations, ils continuent même de goûter, mais ces données qualitatives ne déterminent plus l'interprétation du phénomène. Elles sont disqualifiées. A la limite, le corps devient un parasite de la réaction quand, par exemple, la chaleur du corps de l'expérimentateur influe sur le calorimètre. Le chimiste sensoriel est mort comme sujet historique producteur de savoir. L'évidence sensible, production de lumière, changement de couleur, ne peut rien contre le jugement de la balance, contre les bilans pondéraux qui permettent à Lavoisier d'interpréter ce qui se passe dans la boîte noire de la réaction chimique. Lissa Roberts, à la suite de Golinski, a souligné, à juste titre, que la priorité donnée au verdict des instruments de mesure s'inscrit dans la culture locale de l'Académie royale des sciences, plus généralement de le contexte d'obsession pour les poids et mesures dans la France révolutionnaire.

Il semble donc bien établi, d'une part, qu'il s'agit moins d'une refoulement du corps que d'une déligitimation de sa fontion de sujet de savoir et, d'autre part, que la disqualification du corps est la conséquence de l'essor des intruments, de l'avènement d'une "culture de la précision". Sans contester ces deux thèses, somme toute assez triviales, je voudrais apporter quelques précisions qui compliquent la liaison entre disqualification du corps et promotion des instruments de mesure, J'esquisserai trois remarques.

\section{I) Qu'est ce qu'un instrument dans la chimie du XVIIIe siècle}

La première remarque est une précaution linguistique générale. La thèse du corps supplanté par les instruments dans l'investigation de la nature repose sur une conception présentiste de l'instrument. On a tendance à plaquer sur le XVIIIe siècle notre notion moderne d'instrument comme dispositif technique, maîtrisé, construit dans un but précis d'investigation. L'instrument est "une théorie matérialisée", dira Bachelard, Or au milieu du XVIIIe siècle, mis à part les instruments de physique ou d'optique, les

\footnotetext{
${ }^{1}$ Lissa Roberts, "The death of the sensuous chemist: The new chemistry and the transformation of sensuous technology", Studies in the History and Philosophy of Science, 26, n²4, 1995, pp. 503-529.
} 
instruments ne sont pas les moyens artificiels ou ruses inventés pour interroger la nature, pour la soumettre à nos questions. Cette conception de l'expérience, qui soumet l'objet au projet d'un sujet rationnel, codifiée par Kant à la fin du XVIIIe siècle, me semble obscurcir notre compréhension de la chimie du XVIIIe siècle.

Le concept d'instrument a un sens très particulier dans la chimie du siècle des Lumières. A la suite d'Hermann Boerhaave, la plupart des cours de chimie présentent les instruments de la chimie en six rubriques. Sont instruments les quatre éléments, la terre, l'air l'eau et le feu, puis les menstrues (c'est-à-dire les solvants) puis en dernier lieu seulement les appareils de laboratoire. Qu'est-ce qu'un instrument? Boerhaave le définit ainsi:

"Dans quelque art que ce soit, où l'on se propose de changer les corps; on appelle instrument celui à qui ont peut imprimer, ou auquel on a déjà imprimé un mouvement capable de produire le changement qu'on a en vue" 2 .

L'instrument est un concept beaucoup plus large que notre concept d'appareil conçu en vue d'une fin, qu'on appelait alors machine, et plus proche de la notion aristotélicienne d'organon. Est instrument toute médiation pour l'accomplissement d'un projet, quelle que soit sa fonction. L'instrument n'est pas forcément maîtrisé. Bien que le feu soit réputé l'instrument privilégié du chimiste, sa nature reste enjeu de débats. Boerhaave consacre tout un volume à la nature du feu et, dans les années 1740 , un concours de l'académie soumet cette question aux candidats.

Ce concept d'instrument confondant nos catégories de nature et d'artifice est à rapprocher du concept d'opérations chimiques. Comme l'a très justement remarqué F. Larry Holmes, les historiens de la chimie ont tendance à projeter sur la chimie du XVIIIe siècle notre concept moderne de réaction. Or les chimistes du XVIIIe siècle ne parlent jamais de "réactions chimiques". Ils utilisent plutôt le terme d'opérations chimiques dont ils distinguent deux sortes: unir et séparer ${ }^{1}$. Rien pas même la loi d'attraction de Newton ni les tables de rapports ou d'affinités ne distingue l'action d'une substance sur une autre de l'action du chimiste sur la nature. Les opérations de la nature et de l'art usent des mêmes instruments, des mêmes procédés. La nature elle même est conçue comme un théâtre d'opérations. Elle opère, comme le chimiste dans son laboratoire et met à disposition du chimiste des moyens, les substances naturelles sont autant d'instruments pour l'industrie humaine ou l'investigation de la nature Jean jacques Rousseau, par exemple écrit au sujet des quatre éléments :

${ }^{2}$ Boerhaave, Elémens de chymie, trad du latin, Paris, 1754, T.I p. 267 
"Ces diverses substances en vertu de leurs différents rapports devenant de véritables instruments entre les mains du chymiste pour séparer, réunir, combiner tous les corps simples, mixtes ou composés, qu'il veut soumettre à ses recherches. J'ai cru devoir les compter au nombre des instruments que l'industrie humaine a trouvé le moyen d'appliquer aux travaux de la chimie"3.

Il me semble donc important de souligner que la valorisation du corps comme sujet de savoir s'inscrit dans un cadre où l'homme et la nature forment un tout indistinct où la dichotomie sujet/objet n'est pas privilégiée.

\section{II) La distinction par le corps}

Le corps n'est pas lui-même un instrument de connaissance, pas plus que le thermomètre qui est décrit comme application ou manifestation des propriétés du feu. Quel est donc le rôle ou la fonction du corps dans le discours des chimistes prélavoisiens?

Historiquement, on constate que chaque fois que les chimistes mettent l'accent sur le rôle du corps dans la connaissance, c'est dans une situation polémique et pour affirmer l'identité de la chimie face à d'autres modes de savoir. J'en donnerai deux exemples:

Comme l'a souligné remarquablement John Christie, la rhétorique paracelsienne exalte le corps sujet de connaissance contre le livre, contre l'école, c'est à dire contre la médecine galiénique dominante.

"Laissez moi vous dire ceci : le moindre petit cheveu sur mon cou en sait plus que tous vos scribes; et les boucles de mes chausssures sont plus savantes que vos Galien et Avicenne et ma barbe a plus d'expérience que toutes vos grandes écoles"4.

Ces paroles plus ou moins authentiques suggèrent que le savoir n'est pas localisé où on l'attend et certainement pas dans les livres ni dans les écoles. L'alchimiste est d'abord et avant tout un corps qui voyage qui explore le code de la nature avec ses pieds. Le corps du chimiste est explorateur. Mais en outre, pour défier la culture du livre ou de l'école,

3 voir par exemple, Rousseau J.J. Institutions chimiques, Livre III chap 4 p. 89

${ }^{4}$ Paracelse, Sämlitche Werke, eds Sudhoff and watthiessen (15 vols Munich, Berlin, 1922-33, vol 8 (Paragramum) p. 65; John Christie "The Paracelsian Body". 
Paracelse présente le corps comme un conservatoire de savoir, qui, pareil à une bibliothèque, concentre, emmagasine, capitalise, le savoir.

Le deuxième exemple est tiré de l'Encyclopédie de Diderot et D'Alembert, où le corps du chimiste sert un combat contre le mécanisme triomphant. Dans l'article "chymie", rédigé par Gabriel-François Venel, l'exaltation du corps comme sujet de savoir est un manifeste anti-mécaniste, l'anti-Descartes $^{5}$. Le corps n'est plus une arme tactique contre le livre mais contre l'entendement, l'intellect.

Pour bien comprendre les raisons de cette argumentation, il faut se rappeler la célèbre métaphore du morceau de cire développée par Descartes dans la deuxième de ses Méditations métaphysiques. A la question "qu'estce que connaitre distinctement les corps, la matière", Descartes répondait que seul l'entendement connaît.

"Prenons pour exemple ce morceau de cire qui vient d'être tiré de la ruche : il n'a pas encore perdu la douceur du miel qu'il contenait, il retient quelque chose de l'odeur des fleurs dont il a été recueilli; sa couleur, sa figure, sa grandeur sont apparentes; il est dur, froid, si on le touche, et si vous le frappez il rend quelque son. Or quelle est cette cire, qui ne peut être conçue que par l'entendement ou l'esprit? Certes c'est la même que je vois, que je touche, que j'imagine, et la même que je connaissais depuis le commencement. Mais ce qui est à remarquer, sa perception, ou bien l'action par laquelle on l'aperçoit, n'est point une vision, ni un attouchement, ni une imagination, et ne l'a jamais été, quoiqu'il semblât ainsi auparavant, mais seulement une inspection de l'esprit"6

Descartes souligne le contraste entre le caractérère superficiel et instable de l'approche sensorielle qui croit connaître en mobilisant tous les sens et la profondeur de l'approche intellectuelle, par l'entendement, qui saisit l'essence de la matière- ce qui demeure invariant par changement d'état - c'est-à-dire l'étendue en longueur, largeur et profondeur.

Cette métaphore constitue un défi pour les chimistes car Descartes ne disqualifie pas seulement les sens pour connaître la matière en général (ce qui relève aujourd'hui de la physique) mais pour la connaissance de ce morceau de cire singulier. L'entendement assume la même visée que la physique aristotélicienne. La conclusion de Descartes constituait donc un défi pour les chimistes qui définissent leur discipline comme l'étude des principes constituant les corps de la nature ou de l'art dans leur particularité.

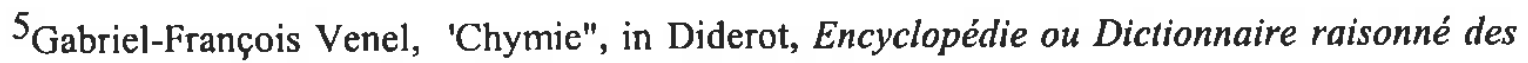
sciences et des arts, T. III, 1753.

${ }^{6}$ Descartes Médilations métaphysiques, 1642, ed Pléiade p. 279-81 
Face à cette prétention à mieux connaître les corps particuliers par "une inspection de l'esprit" que par les sens, face à cette réduction de la matière à une étendue géométrique, face à l'invasion du mécanisme cartésien dans les cours de chimie, Gabriel-François Venel riposte dans une exaltation du corps comme sujet de savoir. Loin d'être un signe de la perfection de la mécanique, la connaissance intellectuelle, l'abstraction et la mathématisation sont, à ses yeux, un écran, un masque du réel. L'abstraction et les agents mécaniques sont des fictions, des apparences d'explication, qui satisfont l'esprit à peu de frais, presque des vertus dormitives.

"Les chymistes ne s'honorent d'aucun agent mécanique et ils trouvent même fort singulier que la seule circonstance d'être éloigné d'un seul de gré de la cause inconnue ait rendu les principes mécaniques si chers à tant de philosophes et leur ait fait rejeter toute théorie fondée immédiatement sur les causes cachées, comme si être vrai n'était autre chose qu'être intelligible, ou comme si un prétendu principe mécanique interposé entre un effet et la cause inconnue, les rassurait contre l'horreur de l'inintelligible"'

Parce que le réel ne se réduit pas à la rationalité mécanique, parce que la nature est complexe, le travail du corps, l'éducation des sens et l'acquisition d'un art sont nécessaires à l'investigation des principes. Venel proclame donc que le "vrai" chimiste doit mettre bas la robe, et se salir les mains.

"C'est la nécessité de toutes ces connaissances pratiques, les longueurs des expériences chimiques, l'assiduité du travail et de l'observation qu'elles exigent, les dépenses qu'elles occasionnent, les dangers auxquels elles exposent, l'acharnement même à ce genre d'occupation qu'on risque toujours de contracter, qui ont fait dire aux chimistes les plus sensés que la chimie était une passion de fou" ${ }^{18}$.

Le savant chimiste fait alliance avec l'artiste ou artisan car il reconnaît que l'avenir de la chimie dépend de "la faculté de juger par sentiment qui s'appelle coup d'oeil chez l'ouvrier, et que celui-ci doit à l'habitude de manier son sujet". Pour être capable de remonter aux principes premiers, le chimiste doit donc mobiliser toutes les ressources humaines. A la raison, il faut ajouter une passion et un art consommé acquis à force de

\footnotetext{
${ }^{7}$ Venel, Encylcopédie, art "Chymie", T. III, p. 415 A

${ }^{8}$ Venel, Encylcopédie, art "Chymie", T. III, 1753. p 421B
} 
patience. Venel demande au chimiste de garder "son thermomètre au bout des doigts et son horloge dans la tête", et juge ridicules toutes les "mesures artificielles" qu'on voudrait substituer à la lecture experte des indices grossiers et sensibles.

\section{III) Le corps qui labore}

Mais à trop exalter les sens au détriment des instruments de mesure, Venel rend-il bien compte des fonctions du corps dans le laboratoire de chimie? En soulignant la singularité de la chimie par la nécessité d'une subversion de la division sociale et intellectuelle entre le chimiste scientifique et l'artisan, Venel fait écho à l'idéal que défendait Diderot dans 1'Interprétation de la Nature. Mieux que Venel cependant, Diderot a su décrire le rôle du corps dans les sciences comme la chimie. Il distinguait, en effet, deux catégories de philosophes : ceux qui "réfléchissent", "ont beaucoup d'idées et n'ont point d'instruments", et ceux qui "se remuent", ont "beaucoup d'instruments et peu d'idées", habituellement méprisés des premiers ${ }^{9}$, Ceux qui se remuent "ont vu si souvent et de si près la nature dans ces opérations", qu'ils en ont acquis "cet esprit de divination par lequel on subodore, pour ainsi dire, des procédés inconnus, des expériences nouvelles, des résultats ignorés". En revanche ceux qui réfléchissent mettent en cuvre les faits, tentent de les lier, "s'opiniâtrant à la solution de problèmes peut-être impossibles" 10 .

Pourquoi les sciences ont-elles fait si peu de progrès?, se demande Diderot. "Parce que les sciences abtraites ont occupé trop longtemps et avec trop peu de fruits les meilleurs esprits" tandis que les"manouvriers de l'expérience", les philosophes ouvriers qui peinent toute leur vie pour sonder la nature passent souvent à côté des découvertes ${ }^{11}$. Aussi Diderot appelle-t-il de ses voeux une "ligue philosophique" entre les deux catégories de philosophes.

Philosophe qui se remue, qui s'agite et labore, c'est à cette catégorie qu'appartient le chimiste,.Le rôle du corps qui caractérise la recherche en ce domaine est le corps au travail, le corps qui fatigue, sue et peine. S'il existe une constante dans l'histoire de la chimie, une permanence qui pourrait constituer l'identité de la discipline chimique à travers les siècles, c'est bien le laboratoire qui la peut définir. Le chimiste est un philosophe ouvrier, un

\footnotetext{
9 . Diderot, De l'Interprétation de la Nature, section 1.

10. Diderot, op. cit, sections 27 à 30 .

$11_{\text {ibid p. } 190}$
} 
travailleur manuel. La notion de labeur, de peine, de travail fatigant est un trait permanent. On le trouve déjà chez Paracelse et Glauber, chez Venel et chez Diderot. Et contrairement au corps sensible, cette configuration du corps au travail n'a pas été effacée par le développement d'une chimie quantitative instrumentale.

Certes Lavoisier prétend, dans le Discours préliminaire au Traité élémentaire de chimie, que sont but est former un chimiste en peu de temps, grâce à une langue bien faite, en procédant à la manière ds géomètres du connu à l'inconnu, Mais cela ne supprime pas, au contraire, le travail de production des faits que l'analyse doit combiner. Le seul écart par rapport au discours de Diderot est que Lavoisier transpose à la chimie le processus de genèse des idées dans la philosophie de Condillac. Il met expérience et observation à la place de sensation. Mais le labeur de l'expérience n'est pas allégé par l'utilisation des instruments au contraire. Il reste tout un travail d'éducation aux manipulations délicates d'appareils de mesure extrêmement sensibles qui n'est pas plus aisé que l'éducation des sens que proposait le cours de Rouelle. Dans la troisième partie du Traité, Lavoisier souligne i) qu'un livre ne remplacera jamais le travail de laboratoire, c'est à dire l'acquisition d'un savoir manipuler (p. 324); ii) que la manipulation des balances est délicate : "Il faut les connâitre, les avoir étudiées, savoir s'en servir, et l'on n'y parvient qu'après un long usage et avec beaucoup d'attention" (p. 335). Là où suffisait le coup d'oeil d'un artiste, le chimiste "moderne" éduqué à la moed Lavoisier, doit faire des pesées répétées, attendre que le fléau revienne à l'équilibre, pour recommencer, iii) Aux lenteurs des manipulations s'ajoutent encore de longs et pénibles calculs pour estimer les résultats, évaluer les erreurs, jongler avec les unités de mesure. Toute une gymnastique arithmétique vient alourdir le travail occasioné par des expériences quantitatives déjà longues et fastidieuses. iv) De plus la balance toute seule ne prouve rien. Elle n'est vraiment démonstrative qu'une fois cuplée avec d'autres instruments de mesure, insérée dans un réseau qui comprend l'aréomètre, le thermomètre, le gazomètre; tous instruments difficiles à manipuler et à standardiser. Lavoisier reconnaît le coût à la fois financier et le coût en énergie de la chimie "moderne". Ainsi conclut-il à propos du gazomètre :

"C'est un instrument précieux par le grand nombre des applications qu'on en peut faire, et parce qu'il est des expériences à peu près impossibles sans lui. Ce qui le renchérit, c'est qu'un seul ne suffit pas, il le faut double dans un grand nombre de cas, comme dans la formation de l'eau, dans celle de l'acide nitreux, \&tc. C'est un effet inévitable de l'état de perfection dont la chimie commence à s'approcher, que d'exiger des instruments et des 
appareils dispendieux et compliqués: il faut s'attacher sans doute à les simplifier, mais il ne faut jamais que ce soit aux dépens de leur commodité et surtout de leur exactitude"12.

Bref Lavoisier n'ignore pas le coût de la précision, il l'ignore si peu qu'il recommande des instruments plus commodes pour les usages sociaux tels que le commerce. Enfin, dans ses mémoires, malgré la rhétorique très codifiée qu'il utilise, faisant l'économie des détails de la campagne d'expérience, Lavoisier confesse parfois la fatigue, le labeur de l'expérimentateur. S'il déclare que seuls les poids et mesures peuvent révéler la nature, permettre d'accèder à la compositiondes substances, Lavoisier ajoute aussitôt que cela exige de la vigueur, que les expériences sont longues et difficiles, si pénibles parfois qu'il termine en renonçant. Ainsi par exemple à la fin du mémoire sur la calcination de l'étain, Lavoisier déclare qu'il manque de courage pour poursuivre ce genre d'expérience ${ }^{13}$.

Loin donc d'éviter le corps au travail, la pratique instrumentale oblige le corps à de nouvelles fatigues, de nouveaux apprentissages et impose toute une économie de la recherche. La pratique instrumentale de Lavoisier n'a pas produit d'effet de rupture dans cette participation fondamentale du corps à l'aventure chimique.

Le corps de l'expérimentateur au travail permet cependant une nouveauté dans la mesure où Lavoisier le prend pour objet de recherche. On connaît le fameux sépéia de Madame Lavoisier représentant les expériences sur la respiration. Seguin le collaborateur de Lavoisier, cosignataire du mémoire, est pris comme cobaye pour étudier la dépense en oxygène d'un travail quelconque. Or ce mémoire est celui-là même où Lavoisier et Seguin suggèrent la possibilité d'une sorte d'unité de mesure de tout travail animal permettant d'évaluer "à combien de livres en poids répondent les efforts d'un homme qui récite un discours, d'un musicien qui joue d'un instrument. On pourrait même évaluer ce qu'il y a de mécanique dans le travail du philosophe qui réfléchit, de l'homme de lettres qui écrit, du musicien qui compose"14. Ainsi le corps sujet du savoir chimique, parce qu'il consomme et produit du travail, s'inscrit dans le cycle d'échanges et de circulation de la nature. Le corps humain instrument de connaissance appartient à la nature, elle-même objet de savoir, exactement

\footnotetext{
12 Lavoisier Traité, $3^{\circ}$ partie, p. 359-60

${ }^{13}$ Lavoisier, Oeuvres T. II, p. 120.

${ }^{14}$ Lavoisier, Oeuvres T. II, p. 698-99
} 
comme les quatre éléments de la nature faisaient jadis partie de l'équipement instrumental du chimiste.

En conclusion, la culture instrumentale n'a pas refoulé le corps du chimiste. Celui-ci est toujours à l'œuvre dans un univers de mesure même si ses fonctions changent. C'est plutôt la promotion du corps qui est à expliquer. Le. corps du chimiste a été parfois brandi comme une arme stratégique pour lutter contre le monopole d'autres instruments de savoir - le livre ou l'entendement - pour promouvoir une épistémologie propre à la chimie.

De plus, le chimiste sensoriel, celui qui est attentif aux couleurs aux odeurs, celui qui goûte, sent, renifle, celui qui a acquis le coup d'oeil n'est pas mort mais simplement soumis au verdict des mesures réputées plus objectives $^{15}$. La balance de Lavoisier et la chimie quantitative instrumentale qui se développe au XIXe siècle n'ont pas profondément ébranlé la conception traditionnelle du laboratoire comme un lieu de travail pénible, fatigant, coûteux. Loin d 'être considéré comme un élément d'imperfection, un résidu opaque d'irrationalité dans la science chimique, ce travail du corps et tout le savoir tacite qu'il enveloppe sont présentés par Lavoisier comme susceptibles d'être mesurés à l'aide de la balance.

Ajoutons enfin que l'analyse qualitative fondée sur les sens n'a jamais disparu de la chimie. Comme le souligne Emile Meyerson, Lavoisier n'a pas débarrassé la chimie de tout ce qui a trait à la qualité"16. Pour la recherche analytique on utilise les qualités multiples des composants. L'analyse qualitative reste très importante et ce n'est pas un hasard si au début du XIXe siècle on a choisi les noms de chlore, chrome pour des substances dont les sels sont très colorés.

Bernadette Bensaude-Vincent

\footnotetext{
${ }^{15}$ Comme chez Descartes, la disqualification de la connaissance sensible au profit d'une démarche intellectuelle plus abstraite correspond à la recherche de ce qui se conserve : la res extensa chez Descartes, la masse chez Lavoisier. Mais cette notion abstraite de conservation de la matière n'est elle-même qu'un instrument, un opérateur entre les mains du chimiste, une condition pour faire marcher la balance pour écrire des bilans de réaction.

${ }^{16}$ E. Meyerson, "Le sens commun et la quantité" (1923), in Essais, Vrin, 1936, p. 18.
} 